Revista de Psicología Vol. 37 (1), 2019 (ISSN 0254-9247)

\title{
La praxis en la formación en Psicología Comunitaria: una mirada ética ${ }^{1}$
}

\author{
Miryam Rivera Holguín², Tesania Velázquez ${ }^{3}$, Elba Custodio Espinoza ${ }^{4}$, \\ Adriana Hildenbrand ${ }^{5}$, Andrea Wakeham ${ }^{6}$ \\ Pontificia Universidad Católica del Perú
}

\begin{abstract}
El objetivo de este estudio fue conocer la praxis en la formación en Psicología Comunitaria, e identificar las características del rol y quehacer ético del psicólogo/a comunitario, a partir del análisis de los sílabos de los cursos de Psicología Comunitaria en las carreras de Psicología que contemplan dichas asignaturas en su plan de estudio en el Perú. Se busca analizar en qué medida el acompañamiento al trabajo práctico se incluye como parte sustancial de la formación del psicólogo/a comunitario. Los resultados señalan que 16 de los 20 sílabos
\end{abstract}

1 Agradecemos a María Inés Winkler, coordinadora del estudio sobre ética en la formación de la Psicología Comunitaria realizado en ocho países miembros de la Red Latinoamericana de Formación en Psicología Comunitaria, por el acompańamiento a este estudio. Esta investigación es parte de un estudio mayor sobre ética en la Formación en Psicología Comunitaria en el Perú, del cual es parte Carolina Vera, a quien agradecemos por sus aportes.

2 Magíster en Salud Mental en Poblaciones por la Universidad Peruana Cayetano Heredia. Docente Asociada del Departamento de Psicología de la Pontificia Universidad Católica del Perú. Dirección postal: Tiensestraat 102-3720, Leuven 3000, Bélgica. Contacto: mriverah@ pucp.pe. ORCID: https://orcid.org/0000-0003-0044-7788

3 Magíster en Psicología Clínica y Forense por la Universidad de Salamanca. Directora de la Dirección Académica de Responsabilidad Social y Docente Asociada del Departamento de Psicología y de la Maestría en Psicología Comunitaria de la PUCP. Dirección postal: Avenida Universitaria 1801, San Miguel, Lima 32, Perú. Contacto: tvelazq@pucp.pe. ORCID: https://orcid.org/0000-0002-7256-8629

4 Magíster en Cooperación al Desarrollo Descentralizado por la Universidad del País Vasco. Docente del Departamento de Psicología y de la Maestría en Psicología Comunitaria de la PUCP. Dirección postal: Avenida Universitaria 1801, San Miguel, Lima 32, Perú. Contacto: custodio.ee@pucp.pe. ORCID: https://orcid.org/0000-0001-7201-6093

5 Licenciada en Psicología Clínica por la Pontificia Universidad Católica del Perú. Contacto: adriana.hildenbrand@pucp.pe. ORCID: https://orcid.org/0000-0001-5015-1809

6 Licenciada en Psicología por la Pontificia Universidad Católica del Perú. Docente del Departamento de Psicología de la Pontificia Universidad Católica del Perú. Dirección postal: Avenida Universitaria 1801, San Miguel, Lima 32, Peru. Contacto: awakeham@pucp.edu.pe. ORCID: https://orcid.org/0000-0003-2739-9659 
de los cursos ofrecidos por las universidades analizadas cuentan con una formación teórico-práctica; pero solo un sílabo da cuenta de espacios de acompañamiento, supervisión o monitoreo de manera explícita. Además, la reflexión sobre el rol y quehacer comunitario está limitada a lo actitudinal y no a la reflexión crítica, perdiéndose la dimensión ético-política de la formación en Psicología Comunitaria.

Palabras clave: praxis, formación, Psicología Comunitaria, ética.

\section{The praxis in training of Community Psychology: An ethical view}

The aim of this study was to explore the praxis in training of Community Psychology and identify the characteristics of the role and ethical work. This was done by analyzing the syllabi of Community Psychology courses in Psychology programs in Peru that include those courses in their plan of studies. The study seeks to analyze the extent of supervision of practical work that is included as a substantial part of the training. The results show that 16 of the 20 syllabi of the courses offered by the analyzed universities included theoretical and practical training, but that only one course explicitly offers a space for accompaniment, supervision and / or monitoring. In addition, there is only reflection on one's attitude toward the role and community involvement, and not critical reflection. Thus, the ethical and political dimension of training in Community Psychology is absent.

Keywords: praxis, training, community psychology, ethics.

\section{A práxis na formaçáo da Psicologia Comunitária: Uma visáo ética}

O objetivo deste estudo é conhecer a práxis na formação da Psicologia Comunitária e identificar as características do papel e do trabalho ético. Isso foi feito analisando os silabos dos cursos de Psicologia Comunitária. Busca também analisar a extensão do acompanhamento ao trabalho prático como parte substancial da formação do psicólogo comunitário. Os resultados mostram que 16 dos 20 currículos dos cursos oferecidos pelas universidades analisadas possuem formação teórica e prática; Mas que apenas um curso oferece um acompanhamento de espaço, supervisão e / ou monitoramento explicitamente. Além disso, a reflexão sobre o papel ea tarefa da comunidade se limita não à reflexão atitudinal e crítica, perdendo a dimensão ética e política da formação em Psicologia Comunitária

Palabras-chave: Praxis, formação, psicologia comunitária, ética

\section{La praxis dans la formation en psychologie communautaire: un regard éthique}

L'objectif de cette étude était de connaître la praxis dans la formation en psychologie communautaire et d'identifier les caractéristiques du rôle et du travail éthique du psychologue communautaire à partir de l'analyse des programmes des cours de psychologie communautaire en carrières de psychologie. Ils envisagent ces sujets dans leur plan d'étude au Pérou. L'objectif est d'analyser dans quelle mesure l'accompagnement au travail pratique fait partie intégrante de la formation du psychologue communautaire. Les résultats indiquent que 16 des 20 syllabes des cours proposés par les universités analysées ont une formation théoriquepratique; mais qu'un seul programme rend compte des espaces d'accompagnement, de supervision ou de surveillance de manière explicite. En outre, la réflexion sur le rôle et le travail communautaire se limite à l'attitude et non à la réflexion critique, perdant ainsi la dimension éthique et politique de la formation en psychologie communautaire.

Mots-clés: Praxis, formation, psychologie communautaire, éthique 
En Latinoamérica ha existido un significativo incremento en la cantidad de universidades, lo cual impacta directamente en la calidad de estudios que se ofrece. Específicamente en el Perú, existen 51 universidades públicas y 92 privadas, producto del nuevo marco de liberalización de la educación superior generado por el decreto legislativo 882, Ley de Promoción de la Inversión en la Educación, promulgada en el año 1996. Ante severos cuestionamientos del nivel de selectividad y calidad de la educación superior privada y pública (Rodríguez y Montoro, 2013), se promulgó a finales de 2014 una nueva Ley Universitaria (ley 30220), que busca garantizar un modelo de calidad para la acreditación de las carreras profesionales, creando la Superintendencia Nacional de Educación Superior Universitaria (Sunedu) (Sineace, 2014). No obstante, son muchas las universidades que aún no cuentan con funcionamiento definitivo, en consecuencia, en el Perú, solo 48 universidades funcionan con Licenciamiento de Sunedu.

En el Perú, la carrera de psicología se ofrece desde el año 1955 en Lima (Livia, 2014); en años posteriores y sobre todo a comienzos del presente siglo, se comenzó a dictar esta carrera en regiones, principalmente en universidades privadas. Hacia 2015, se encuentra que 42 universidades ofrecían formación en Psicología en el Perú (7 públicas y 35 privadas). Los espacios formativos de psicología en el Perú están dirigidos a estudiantes de pregrado y posgrado y buscan acompañar, supervisar y evaluar el proceso de adquisición de conocimiento y desarrollo de competencias, pero se ha identificado que está débilmente vinculada a los problemas psicosociales de la sociedad (Anicama, 2010).

Si bien se ha desarrollado una línea de profesionalización, aún falta trabajar y estimular la coproducción de conocimiento y la mirada reflexiva acerca de las propias implicancias en la tarea de atender y cuidar a la población que lo demande (Gutiérrez, 2005). La apuesta es lograr una formación con posibilidad de aprender, escuchar, analizar 
y fortalecer el saber hacer, desde el respeto a las diferencias, atravesados por incertidumbres y falta de certezas (Fernández, 2006).

Por su parte, la formación en Psicología Comunitaria permite cuestionar la teoría para adaptarla a una realidad, mediante la praxis, en el que se hace la teoría y se piensa la práctica (Ussher, 2008). En esta tarea de aprender desde la práctica, el acompañamiento requiere una dimensión reflexiva que aporta a recrear prácticas y teorías; además, permite el reconocimiento de los múltiples contextos sociales, culturales y políticos como ámbitos en los que se construye y de-construye la identidad, el mundo emocional, experiencial y explicativo, los cuales forman parte de la realidad que se vive y que son, al mismo tiempo, susceptibles de transformación (Brenes, Burgueño, Casas \& Pérez, 2010; Rivera-Holguín, Velázquez, Custodio \& Corveleyn, 2018).

Asimismo, la formación académica de los/as psicólogos/as comunitarios requiere que la formación sea teórico-práctica, para que sea coherente con los principios y la apuesta política y ética de la Psicología Comunitaria (Nelson \& Prilleltensky, 2005; Bess, Fisher, Sonn \& Bishop, 2002). De esta manera, la formación permitirá analizar desde diferentes frentes las relaciones de poder que se ejercen entre los múltiples actores involucrados en el ejercicio preprofesional. Esta reflexión permite visibilizar que muchas veces los actores se mueven bajo lógicas distintas, inscribiéndose en líneas éticas diferentes a las que se brindan en la academia; por ello, los futuros psicólogos comunitarios necesitan de estos espacios de acompańamiento que les permitan establecer un diálogo entre estas diferentes formas de actuar que también influyen en el propio desarrollo de la disciplina psicológica (Winkler, Alvear, Olivares \& Pasmanik, 2014).

En el trabajo del psicólogo comunitario en contextos de desigualdad y pobreza se hace necesario un acompañamiento que permita la planificación, el desarrollo de actividades y la reflexión continua en los momentos de familiarizarse, insertarse, desarrollar una actividad, devolver y cerrar los trabajos psicológicos. En consecuencia, es importante incluir dentro de la formación los espacios de acompañamiento, supervisión y monitoreo. En ellos no solo se debería abordar 
una problemática puntual, sino también el contexto, y aquí se hace imprescindible considerar que los actores locales se constituyen en el eje del trabajo comunitario; por lo cual, también aparecen las dinámicas interpersonales como elementos de análisis, abordando los conflictos intersubjetivos, intragrupales e intercomunales, favoreciendo la interdisciplinariedad y la mirada intercultural (Rivera-Holguín y Velázquez, 2015). Esto permite que el futuro profesional incorpore el ejercicio de autorreflexión en su práctica profesional y asuma como precepto ético: "ante todo, no dañar", es decir, reconocer que cada vez que nos acercamos a las comunidades existe la potencial situación de generar acciones que puedan no resultar beneficiosas para la comunidad (IASC, 2007).

El acompañamiento es el seguimiento que se realiza durante la ejecución de la praxis comunitarias a través de las visitas periódicas, lo que permite revisar que los procesos, actividades, resultados e impactos de acuerdo a los objetivos propuestos (Di Virgilio y Solano, 2012). Este proceso posibilita construir desde diferentes escenarios las modalidades y ámbitos de intervención, textos y contextos, espacios y participantes (Fernández, 2007). En este espacio, el cuidado supone respeto a la autonomía, a la autogestión, a lo diferente.

Pero se trata no solo de cuidar a la población con la que se trabaja, sino también a los equipos de profesionales, promotores o estudiantes. Como señalan Velázquez, Rivera-Holguín y Custodio (2015), esta práctica se ha desarrollado a partir del trabajo comunitario en contextos caracterizados por pobreza, exclusión y violencia. En esta medida, el saber está ligado al hacer y también al poder. Las sociedades definen la legitimación de sus saberes a través de mecanismos que encierran conflictos: en ocasiones no se entiende que los diferentes saberes y prácticas permiten generar complementariedad (Wiesenfeld, 2000). En síntesis, un trabajo de Psicología Comunitaria constituye un desafío a las redes de poder, las prácticas y los saberes no solo de los pobladores de la comunidad, sino de los propios agentes comunitarios. Por eso, son frecuentes los conflictos, las negociaciones y las nuevas articulaciones entre los intereses y los afectos de todos los involucrados. 
Montero (2006) plantea que se deben utilizar distintas estrategias que permitan agregar dimensiones que durante la ejecución de la tarea no se visibilizan por los profesionales; se trata de analizar el impacto de las situaciones concretas en cada profesional y en sus vínculos interpersonales. Esta mirada vislumbra que muchas veces en los estudiantes se coloca la impotencia de la población, lo que puede generar parálisis al sentirse abrumados por macroestructuras difíciles de cambiar, que pierdan la perspectiva de lo que sí se puede hacer desde su rol y la misión de la institución de la que forman parte. La universidad es un espacio formativo y de reflexión que debe favorecer a que los estudiantes conozcan la realidad y sean capaces de cuestionarla para generar propuestas en la búsqueda del bien común. En este sentido, consideramos que en Latinoamérica el papel de las universidades no es solamente formar ciudadanos, sino ser agentes de cambio social (Velázquez, 2016).

Sánchez (2004) señala que es importante construir un marco de acuerdo y debate que toma aspectos valorativos, posiciones ideológicas, miradas políticas ya que no se puede trabajar en una comunidad, sin tomar en cuenta las tensiones políticas, la existencia de redes, el devenir histórico, los planes sociales que lo atraviesan, los intereses que se ponen en juego en las acciones colectivas. Al decir de Martín-Baró (2006) se trata de

...que no sean los conceptos los que convocan a la realidad, sino la realidad la que busca los conceptos; que no sean las teorías las que definen los problemas de nuestra situación, sino que sean esos mismos problemas los que reclaman y, por así decirlo, elijan su propia teorización (p. 314 ).

Esta tarea está sostenida en una ética del cuidado, como un proceso que adquiere una doble responsabilidad: cuidar a la persona, grupo, institución y/o comunidad con la cual el profesional trabaja y, también, procurar el cuidado del psicólogo que opera en esos ámbitos. El cuidado que puede manifestarse en espacios de acompańamiento, supervisión y/o monitoreo es parte del desempeño ético de los 
La praxis en la formación en Psicología Comunitaria: una mirada ética / Rivera et al.

profesionales, promotores o estudiantes. Esto respalda la seguridad de la integridad personal, la reflexión crítica, la identificación y atención a los dilemas éticos y formación contextualizada para los miembros de los equipos (Ruiz-Casares, 2014). Por ello, la creación y mantenimiento de espacios de reflexión se vuelve prioritario para reconocer que estamos implicados en el proceso de acompañamiento. Este ámbito está integrado por todas aquellas actividades que promueven una mirada reflexiva, del agente social y de la organización que brinda la atención, sobre su quehacer y las premisas desde las que delinean la relación que construyen con la población con la que se trabaja (Winkler, Alvear, Olivares \& Pasmanik, 2010).

En este sentido, la inclusión de la ética desde la etapa de la formación en Psicología Comunitaria es una responsabilidad, tal como el respeto, la confidencialidad, la beneficencia, el no hacer daño y demás principios éticos de cualquier investigación o intervención en psicología (Leaning, 2001; Collogan, Tuma, Dolan-Sewell, Borja \& Fleischman, 2004; Ruiz-Casares, 2014).

El trabajo con poblaciones en situaciones de vulnerabilidad exige el desarrollo de una ética consiente de las implicancias e impactos de nuestras intervenciones, a partir de un balance entre riesgos, daños y beneficios (Ruiz-Casares, 2014). Este aspecto es fundamental al pensar la formación, diseñar los cursos y organizar la praxis de los futuros psicólogos comunitarios. En este sentido, surge el reto de encontrar un equilibrio entre los tiempos de las comunidades y los tiempos de la academia (Montero \& Giulianin, 1999). Es importante trabajar de forma continua con los grupos y las comunidades, para lo cual el vínculo deberá plantearse desde y con la institución, de manera que se faciliten los procesos y se evite que cada curso y cada semestre empiece con una comunidad "nueva" o que el trabajo práctico se haga con una plantilla de talleres a implementar de manera rígida en las distintas comunidades. Por el contrario, el trabajo continuo permitirá dar valor a los procesos, tanto de aprendizaje, como de familiarización y, sobre todo, de respeto a la comunidad y a los estudiantes. Esa continuidad, al mismo tiempo, será una condición que respalde proyectos sostenibles económicamente. 
Es a partir de esta revisión en la que se evidencia que la ética permite unir la realidad con la producción académica y la praxis (Vera, 2015). La dimensión ética implica no solo un reconocimiento de la alteridad, sino una comunicación horizontal plena: una actitud de apertura a conocer con el otro y esto constituye una deconstrucción radical de las relaciones jerárquicas que colocan la autoridad del saber de la universidad y de sus miembros en una posición privilegiada frente a la comunidad. Esta deconstrucción será facilitada, entre otros, en los espacios de cuestionamiento e intercambio, que han de ser incorporados desde la etapa formativa de los estudiantes.

Partiendo de esta premisa, el presente estudio indaga en la dimensión ética en los cursos de Psicología Comunitaria de pregrado, a partir de la revisión y análisis de los sílabos de los cursos que se dictan en las universidades del Perú. Este análisis focalizado se propone también alcanzar otro propósito: contribuir a la comprensión del rol y el quehacer ético del psicólogo comunitario a partir del trabajo práctico. Para ello, nos planteamos los siguientes objetivos específicos: a) identificar la inclusión de la praxis en la formación en Psicología Comunitaria, b) identificar en qué medida el acompańamiento al trabajo práctico se incluye como parte sustancial de la formación del psicólogo comunitario en el Perú, c) analizar cómo se construye y desarrolla el rol y quehacer del psicólogo/a comunitario a partir del trabajo práctico.

\section{Método}

La presente investigación utiliza una aproximación predominantemente cualitativa, tiene un diseño exploratorio y descriptivo, que a través de la revisión y análisis de los sílabos de los cursos de Psicología Comunitaria, busca identificar las características de la praxis y de los procesos formativos que acompańan la praxis como parte de la formación profesional en psicología comunitaria en las universidades de Perú. 
La praxis en la formación en Psicología Comunitaria: una mirada ética / Rivera et al.

\section{Participantes}

De las 42 universidades que enseñan la carrera de psicología, fueron 18 universidades participantes que autorizaron de manera explícita su disposición de participar en este estudio, a través de una carta de consentimiento firmada. De esta manera, las instituciones participantes de este estudio representan el $42.8 \%$ de universidades que forman psicólogos en el país, y cuenta con variabilidad en cuanto a su carácter público o privado y de ubicación geográfica. Del total de las universidades que participaron en la investigación, se obtuvieron los 20 sílabos del curso de Psicología Comunitaria que se han utilizado para el presente análisis. Cabe resaltar que el proyecto de investigación del que forma parte este estudio fue aprobado por el Comité de Ética para la Investigación con seres humanos y animales de la Pontificia Universidad Católica del Perú.

\section{Técnicas de producción y análisis de datos}

En este estudio se aplicó como estrategia de producción y análisis de datos una aproximación cualitativa, a partir de categorías previas y emergentes, a las cuales se aplicó posteriomente un análisis de frecuencias. Como parte de una investigación internacional, en este estudio se ha utilizado los instrumentos propuestos por Winkler (2007) en la investigación sobre formación en Psicología Comunitaria en Chile. La adaptación del instrumento, una ficha de datos sobre el curso de Psicología Comunitaria, fue realizada en coordinación con la autora original y fue revisada por pares internacionales.

La ficha propuesta incluye una sección de datos generales sobre la universidad y sobre el curso a analizarse, y otra sección cuyas categorías corresponden a la presencia de trabajo de campo, enfoques comunitarios y metodología del curso. El presente trabajo profundiza en los resultados concernientes a las características del trabajo de campo del curso presentadas en los sílabos del curso de Psicología Comunitaria, con énfasis en la metodología y los aspectos éticos. 


\section{Procedimiento}

Una vez identificadas las universidades que forman profesionales de psicología en el Perú y que incluyen en sus planes de estudio cursos de Psicología Comunitaria, se les solicitó autorización para la revisión de los sílabos del curso de tales cursos. Una vez autorizada la revisión, se analizaron 20 sílabos de este curso y se organizó la información de acuerdo a las categorías del instrumento y a las subcategorías que emergieron del ordenamiento de la información. Se aplicó análisis intersubjetivo de la categorización de los sílabos en la ficha a cargo de tres de las investigadoras. Así también, se incluyó el dialogo con los/as investigadores/as de los otros países participantes del estudio internacional (Winkler, Velázquez, Rivera-Holguín, Castillo, Rodríguez, Ayala, 2016).

\section{Resultados y Discusión}

Se analizó cada uno de los sílabos en función de las secciones de la ficha, las cuales permitieron conocer, las características del curso y de la universidad en la cual se dicta y, las características del trabajo de campo. A continuación, presentamos en primer lugar las características del curso y la universidad en la cual se dicta.

De los 20 sílabos de cursos de Psicología Comunitaria, 12 pertenecen a las universidades de Lima y ocho a universidades ubicadas en las diferentes regiones del Perú. En relación al tipo de acceso, se encuentra que, del total de 20 cursos de Psicología Comunitaria identificados, 17 se dictan en universidades privadas y tres de ellos en universidades nacionales. Respecto de los créditos asignados a cada uno de los cursos en los sílabos encontramos variabilidad: de dos a ocho créditos. Cabe señalar que la mayoría de los cursos cuenta con tres créditos, según lo referido en el sílabo (Ver apéndice 1).

A partir de la revisión de los sílabos se encuentra que 16 de las universidades ofrecen el curso como obligatorio y cuatro, como electivo. Este resultado permite reflexionar sobre los constantes cambios sociales, 
no solo las necesidades colectivas cambian, sino también el abordaje que se asume desde la academia. Desde la formación de los futuros psicólogos del país, se busca que los profesionales desarrollen estrategias para responder a los problemas sociales presentes en contextos de alta vulnerabilidad y de desarrollo social como: violencia, exclusión, pobreza, inequidad, entre otras (Seidman y Cappella, 2017). En consecuencia, se observa que desde la formación en psicología ya no solo se está considerando priorizar respuestas a un dolor psíquico individual, sino que incorpora el malestar grupal, donde la comunidad aparece como un actor importante. De esta manera, las intervenciones dependerán también de propuestas comunitarias e interdisciplinarias. Desde la Psicología Comunitaria se busca atender a esta demanda, ya que el desarrollo y fortalecimiento de las capacidades y recursos de las comunidades constituyen el eje principal de la disciplina para promover el desarrollo y fortalecimiento de la sociedad civil y la ciudadanía (Montero, 2010).

En segundo lugar presentamos lo referido al trabajo de campo, 16 de los 20 sílabos analizados cuentan con una formación que incluye énfasis en teoría y práctica; los otros cuatro se imparten solo teóricamente. De los sílabos que dan cabida al trabajo de campo, diez corresponden a universidades en Lima y seis, a regiones.

La Psicología Comunitaria implica un desarrollo de conocimiento conjunto con los actores sociales y el contexto que rodea al grupo o comunidad, pues esta se funda en un constante intercambio, resignificación de la información y generación de nuevo conocimiento a partir del diálogo entre la comunidad y el agente externo (Montero, 2006; Ferullo, 2006). En este sentido, resulta esencial la inclusión del vínculo con la realidad y la praxis en la formación en Psicología Comunitaria, lo cual se reconoce en los 16 sílabos que integran una enseńanza teórico-práctica, que evidencia una apuesta de las universidades por salir del claustro universitario. Los otros cuatro, sin embargo, plantean una enseñanza únicamente desde la teoría. En estos casos, nos preguntamos en qué medida -y de qué manera- es posible transmitir las bases de la Psicología Comunitaria, ya que un diálogo franco y abierto entre teoría y praxis es uno de los mayores retos para esta subdisciplina. 
La forma de plantear el trabajo práctico varía entre los distintos programas. Entre las propuestas, se encuentran los que buscan "una visión amplia sobre los procesos nacionales y locales en curso, en los que se inscribe la práctica comunitaria (...) [y fomentar] capacidad para identificar y solucionar problemas en contextos cambiantes" (sílabo de universidad privada ubicada en Lima), lo cual nos hablaría de poner en diálogo el aprendizaje desde la academia con la retroalimentación del entorno. Sin embargo, también se identificaron sílabos en los que el objetivo del trabajo de campo es una "forma de transmisión de valores" (sílabo de universidad privada ubicada en regiones), donde la población es referida como "muestras seleccionadas" (sílabo de universidad privada ubicada en Lima) o como "objeto de intervención" (sílabo de universidad privada ubicada en regiones), con lo cual se transmite una aproximación jerárquica, donde los participantes de la intervención se entienden como agentes pasivos. De esta manera, el logro que implica la inclusión del trabajo de campo para el diálogo entre la teoría y la praxis puede no siempre alinearse con los planteamientos de la Psicología Comunitaria.

$\mathrm{Si}$ bien es cierto, que se reconoce un interés por vincularse directamente con la realidad y el trabajo de campo, este no viene acompañado de un componente reflexivo, ya que en solo un sílabo se incorpora espacios de acompańamiento, supervisión y/o monitoreo de manera explícita y planificada, otorgando dos sesiones del calendario académico exclusivamente a esta actividad. Es decir, si bien la mayoría de cursos de Psicología Comunitaria incorpora la dimensión práctica en la formación, es difícil encontrar en estos el espacio para llevar de manera conjunta procesos de reflexión que permitan cuestionar los avances y retos del trabajo realizado, así como tomar distancia de funciones asistencialistas y aproximarse al camino de la transformación social.

La discusión y reflexión crítica permite generar aprendizajes mutuos, y así se reconoce los saberes que las comunidades pueden aportar a la formación de los estudiantes. Es necesario recalcar que tener estos espacios de discusión permite identificar, pensar y analizar lo que sucede en su entorno, desde la perspectiva de su propia historia, contextos y 
La praxis en la formación en Psicología Comunitaria: una mirada ética / Rivera et al.

grupos organizados (Montero, 2010; Velázquez, Rivera-Holguín, y Custodio, 2017). De este modo se instala un modo de comunicación horizontal, afectivo y crítico que enriquece a ambos polos de la relación (Rivera-Holguín, et. al. 2018). En once sílabos se reporta que el trabajo de campo es revisado a través de informes o bitácoras que reemplazan el espacio de diálogo y construcción de conocimiento a partir de la reflexión suscitada por el encuentro con la comunidad. Si bien, es posible que estos trabajos permitan un acercamiento a la experiencia vivida, no hay información explícita respecto a la discusión académica respectiva. En los cuatro sílabos restantes no se explicita ni el acompañamiento, ni la presentación de trabajos relacionados a la praxis.

Entonces, la pregunta clave es cómo se está formando al estudiante en el quehacer comunitario, ya que como señala Montero (2004), en la formación de los psicólogos/as comunitarios, es esencial el carácter teórico-práctico, ya que la teoría de la Psicología Comunitaria se elabora desde la praxis y la reflexión de la misma. En ese sentido, la formación exige la inclusión de la praxis y un acompañamiento, supervisión y/o monitoreo que permita una reflexión crítica, un análisis del proceso que incluya logros, retos, encuentros y desencuentros de intersubjetividades, dilemas éticos, y otros aspectos centrales del quehacer comunitario. El trabajo en Psicología Comunitaria implica pensar la praxis y pensar(nos) conjuntamente en el encuentro con la comunidad. Es decir, se aprende en el hacer y en su reflexión. Formar en el quehacer comunitario, es una apuesta ética, que no es posible sin la praxis, la reflexión y el acompańamiento de la misma (Nelson \& Prilleltenski, 2005). La reflexión y el acompañamiento de la praxis, son espacios que dan la posibilidad de aprender, analizar y fortalecer el saber-hacer a partir del diálogo (Fernández, 2006). Como propone Montero (2004):

(...) la reflexión crítica debe acompañar siempre la formación y la praxis comunitaria. Cada sesión de trabajo con la comunidad, debe ser analizada, a fin de proponer en evidencia los aciertos y los errores, el porqué de ciertas conductas y, sobre todo, el cómo aprender de unos y de otras, a la vez que aprendemos a conocer nuestros acuerdos y nuestros rechazos (p. 93). 
Los espacios de acompañamiento, supervisión y/o monitoreo permiten el análisis del contacto con diferentes ámbitos en los que se construye y de-construye los conceptos, la relación y los afectos involucrados (Brenes et al., 2010). Por encontrarse los estudiantes en etapa de formación, resulta necesario incluir estos espacios para pensar en las diferentes formas en las cuales se interactúa con la comunidad, y de qué manera estas impactan en los estudiantes y también en las comunidades, en las instituciones y en el propio desarrollo de la Psicología Comunitaria (Winkler et al., 2014; Rivera-Holguín et. al. 2018). Por ello, resulta importante incluir estos espacios de acompañamiento, supervisión y/o monitoreo dentro de la formación, de manera que el trabajo en campo no solo se piense en la entrega de un informe o reporte orientado a la evaluación del desempeño del estudiante, sino que siga la propuesta comunitaria, que implica, más que el abordaje de una problemática puntual, pensar en una intervención desde la complejidad de un contexto intercultural y favoreciendo la interdisciplinariedad (Fernández, 2006; Rivera-Holguín y Velázquez, 2015). Es importante señalar que estos espacios permitirán al docente y al estudiante analizar la intervención y recordar el principio ético de ante todo no dañar, que, como señalan Winkler et al. (2010), está ligado no solo a la praxis, sino a la reflexión de la misma (Velázquez et. al., 2017).

En este sentido, también es importante que en el marco del trabajo de campo, que se propone como parte de la formación del psicólogo/a comunitario, se analicen las relaciones de poder que existen en el contacto con la población, sobre todo porque en ocasiones lo diferente se desvaloriza para enaltecer al conocimiento científico y académico (Wiesenfeld, 2000). Asimismo, resulta importante la reflexión en torno a las emociones de los estudiantes con respecto a las actividades realizadas. Por ejemplo, es posible que se sientan abrumados por las situaciones que no pueden cambiar; en estos casos, es importante un acompańamiento que permita recuperar la perspectiva de lo que sí se puede modificar, así como del rol de cada uno dentro del trabajo que se realiza (Velázquez, Rivera-Holguín, y Custodio, 2015). Y más aún considerando que la Psicología Comunitaria busca el cambio social que 
La praxis en la formación en Psicología Comunitaria: una mirada ética / Rivera et al.

permita la inclusión y transformación social y cuestiona estructuras sociales verticales que mantienen el status quo (Montero, 2004).

Este proceso motiva la reflexión sobre algunos de los principios de la Psicología Comunitaria, como la participación, que es uno de los ejes comunes de las intervenciones comunitarias. La participación permite transformar los espacios de convivencia y el sentido de comunidad, así como fortalecer capacidades individuales y comunitarias (Rivera-Holguín, Velázquez \& Morote, 2014). Las transformaciones no solo se dan en la comunidad, sino también en los agentes externos que participan de esta dinámica. La ausencia de un contacto directo con la población puede llevar a los estudiantes a ignorar los alcances y retos de la participación; recordemos que desde la Psicología Comunitaria, el establecimiento de un vínculo con el otro es lo que influye en el compromiso, la responsabilidad y la sostenibilidad de las acciones emprendidas (Reich, Riemer, Prilleltensky \& Montero, 2007). Por lo tanto, promover y lograr esa participación comprometida en las relaciones y el espacio privado, así como en el espacio colectivo de la comunidad y en el espacio colectivo-público es un objetivo inmediato dentro de la finalidad más amplia de la transformación (Montero, 2010).

En el análisis de los sílabos, consideramos fundamental el compromiso asumido por la institución universitaria con las estrategias de formación. Si bien, es posible que los docentes incorporen acciones o estrategias de enseńanza adicionales que fortalezcan el aprendizaje de los estudiantes a partir de la praxis, es importante reconocer que estas son iniciativas que no son exigidas desde la institución, con lo cual se puede entender que no estarían siendo consideradas indispensables para una óptima formación de los estudiantes. Son, por el contrario, iniciativas de algunos docentes, muchos de los cuales no cuentan con el soporte institucional. En este sentido, resaltamos como responsabilidad de las universidades la necesidad de establecer un vínculo y un compromiso con los grupos, instituciones o comunidades, así como exigir y facilitar a sus docentes el acompañamiento del trabajo práctico, con el fin de lograr una formación integral y coherente de los estudiantes (Ussher, 2008). 
Lo central del trabajo de campo y de los espacios de acompañamiento, supervisión y/o monitoreo, es que están ligados al rol del psicólogo comunitario. Es decir, se aprende en el hacer, en el conocer y en el encuentro con la comunidad. Enseñar el rol y quehacer comunitario, lo que significa la dimensión ética y política, resulta imposible sin la praxis y el acompañamiento de la misma (Montero, 2006, Winkler et al., 2016). Los estudiantes comprenderán el rol del psicólogo/a comunitario en campo y a partir de los espacios de acompañamiento, supervisión y/o monitoreo, se pensarán y reflexionarán sobre lo que se hizo en el encuentro con la comunidad y con sus equipos de trabajo, asimismo, tales espacios posibilitan que el/la estudiante se den cuenta del valor del cuidado del personal que trabajo en el campo (Velázquez et al., 2015).

En los sílabos revisados, se encuentra que el rol y quehacer comunitario se asocian, en la mayoría de casos, a la escala de valores y la moral de los/as estudiantes, y lo ético se equipara a la deontología y a lo normativo más que a la dimensión política y a la transformación social (Montero, 2006; Winkler, 2007). La deontología está ligada al comportamiento y al hacer profesional; si bien es importante, no basta en los procesos formativos. En este sentido, se identifica una débil inclusión de contenidos o competencias asociadas al reconocimiento de saberes en la comunidad, al aprendizaje colaborativo, a la co-construcción del conocimiento y al respeto e inclusión de las diferencias y a favorecer la participación de la comunidad (Nelson \& Prilleltenski, 2005). Este énfasis en los valores antes que en su aplicación en el campo, se refleja en las preocupaciones de los estudiantes al entrar en contacto con las poblaciones, se ha identificado que relacionan la ética más sus propias ansiedades que con los retos del encuentro con las comunidades (Rodríguez, de la Cuesta, Recto \& Mosquera, 2016).

Finalmente, al analizar lo reportado en los sílabos de cursos de Psicología Comunitaria, se puede concluir que la subdisciplina ha ganado un espacio en el campo de la de la psicología, pero que la formación que se brinda en el Perú se encuentra todavía en una fase inicial. Si bien existe un reconocimiento de articulación teóricopráctica, aún hay limitaciones en la comprensión de la naturaleza de 
dicha interacción y en la necesidad de espacios de reflexión, supervisión y/o monitoreo. Estas limitaciones afectan el aprendizaje óptimo de la Psicología Comunitaria en las universidades peruanas y disminuyen el impacto de su carácter ético-político.

Reconociendo que la Psicología Comunitaria no es una entidad monolítica, sino más bien está conformada por un conjunto de principios, teorías y valores se hace relevante subrayar la necesidad de espacios de supervisión, acompańamiento y/o monitoreo, dado que las necesidades de las comunidades varían alrededor del mundo y se hace inimaginable una formación que reposa solo en la teoría y no en la reflexión de la praxis. Este trabajo responde a la necesidad de reflexionar sobre el desarrollo de la Psicología comunitaria y de la formación con impacto social (Prilleltenski, 2014). Siendo el Perú un país diverso y marcado por la exclusión y discriminación social se requiere de profesionales que sean sensibles a las diferentes necesidades del país, y capaces de construir conocimiento a partir de ello. Los espacios de acompañamiento, supervisión y/o monitoreo posibilitan la co-construcción de conocimiento situado y a su vez preservan el saber-hacer de los profesionales en Psicología Comunitaria y puede contribuir a un marco ético discernible (Campbell, 2016). Por tanto, los resultados de este estudio nos demuestran los primeros pasos en esa ruta.

\section{Referencias bibliográficas}

Anicama, J. (2010). La formación profesional, identidad y metas educacionales del psicólogo en el Perú. Revista Peruana de Psicología, 9(1), 13-23.

Asamblea Nacional de Rectores (ANR) (2012). Datos estadísticos universitarios. http://200.48.39.65/doc/ESTADISTICA_UNIVERSITARIAS.pdf. Fecha de consulta: 09 de noviembre de 2013.

Bess, K., Fisher, A., Sonn, C. \& Bishop, B. (2002). Psychological Sense of Community: Theory, Research, and Application. In A. Fisher, C. Sonn \& B. Bishop (Eds.), Psychological Sense of Community: 
Research, Applications and Implications (pp. 3-22). Nueva York: Kluwer Academic/Plenum Publisher.

Brenes, A., Burgueño, M., Casas, A. \& Pérez, E. (2010). José Luis Rebellato, intelectual radical. Montevideo: EPPAL, Nordan y Extensión.

Campbell, R. (2016). "It's the way that you do it": Developing an ethical framework for community psychology research and action. American Journal of Community Psychology, 58(3-4), 294-302. https://doi.org/10.1002/ajcp.12037

Collogan, L., Tuma, F., Dolan-Sewell, R., Borja, S. \& Fleischman, A. (2004). Ethical Issues Pertaining to Research in the Aftermath of Disaster. Journal of Traumatic Stress, 17(5), 363-372. https://doi. org/10.1023/B:JOTS.0000048949.43570.6a

Creswell, J. (2007). Qualitative Inquiry \& Research Design. Choosing among five approaches. Thousand Oaks: Sage Publications.

Di Virgilio, M. \& Solano, R. (2012). Monitoreo y evaluación de políticas, programas y proyectos sociales. Buenos Aires: CIPPEC y UNICEF. Fernández, A. (2006). Politica y subjetividad. Buenos Aires: Tinta Limón.

Ferullo, A. (2006). El triángulo de las tres "P". Psicología, participación y poder. Buenos Aires: Editorial Paidós.

Gutiérrez, O. (2005). Educación y entrenamiento basados en el concepto de competencia: implicaciones para la acreditación de los programas de psicología. Revista Mexicana de Psicología, 22, número monográfico especial, 253-270.

Interagency Standing Committee (IASC) (2007). IASC Guidelines in Mental Health and Psychosocial Support in Emergency Settings. Ginebra: IASC.

Leaning, J. (2001). Ethics of Research in Refugee Populations. The Lancet, 357(5), 1432-1433. https://doi.org/10.1016/S01406736(00)04572-4

Livia, J. (2014). El desarrollo de la psicología en el Perú. Información Psicológica, (108), 45-58. 
La praxis en la formación en Psicología Comunitaria: una mirada ética / Rivera et al.

Martínez, M. (2006). La investigación cualitativa (síntesis conceptual). Revista IIPSI Facultad de Psicología UNMSM, 9(1), 123-146.

Martín-Baró, I. (2006). Psicología de la liberación. Madrid: Trotta.

Montero, M. (2004). Introducción a la Psicología Comunitaria. Buenos Aires: Paidós.

Montero, M. (2006). Hacer para transformar. Buenos Aires: Paidós.

Montero, M. (2010). Fortalecimiento de la ciudadanía y transformación social: Área de encuentro entre la Psicología Política y la Psicología Comunitaria. Psykhe (Santiago), 19(2), 51-63. https://doi.org/10.4067/S0718-22282010000200006

Montero, M. \& Giuliani, F. (1999). La docencia en la psicología social comunitaria: algunos problemas. Psykhe, 8(1), 57-63.

Nelson, G. \& Prilleltensky, I. (Eds.) (2005). Community Psychology. In Pursuit of Liberation and Well-being. Nueva York: Palgrave Macmillan. https://doi.org/10.1007/978-0-230-21400-2

Prilleltensky, I. (2014). Meaning-making, mattering, and thriving in community psychology: From co-optation to amelioration and transformation. Psychosocial Intervention, 23(2), 151-154. https://doi.org/10.1016/j.psi.2014.07.008

Reich, S. M., Riemer, M., Prilleltensky, I. \& Montero, M. (2007). International Community Psychology: Theory and Histories. Nueva York, NY: Springer. https://doi.org/10.1007/978-0-387-49500-2

Rivera-Holguín, M., Velazquez, T. \& Morote, R. (2014). Participación y fortalecimiento comunitario en un contexto posterre-moto en Chincha, Perú. Revista Psicoperspectivas 13(2),144-155.

Rivera-Holguín, M. \& Velázquez, T. (Eds.) (2015). Trabajo con personas afectadas por violencia politica: Salud mental comunitaria y consejería. Lima: Maestría en Psicología Comunitaria PUCP, UARM, UKL.

Rivera-Holguín, M., Velázquez, T., Custodio, E. \& Corveleyn, J. (2018). Improving community mental health services for people affected by political violence in Ayacucho, Perú, Journal of Prevention \& Intervention in the Community, 46(1), 100-112. https://doi.org/10.1080/10852352.2018.1386352 
Rodríguez, J. \& Montoro, L. (2013). La educación superior en el Perú: situación actual y perspectivas (No. 2013-370). Lima: Departamento de Economía, Pontificia Universidad Católica del Perú.

Ruiz-Casares, M. (2014). Research Ethics in Global Mental Health: Advancing Culturally Responsive Mental Health Research. Transcultural psychiatry, 51(6), 790- 805.

Sánchez, A. (2004). Acción social en tiempos de conformismo: por una ética posible de la intervención comunitaria. En A. Sánchez, A. Zambrano y M. Palacín (Eds.), European Community Psychology: Community, Power, Ethics and Values (pp. 93-106). Barcelona: Publicacions Universitat de Barcelona.

Seidman, E. \& Cappella, E. (2017). Social settings as loci of intervention. Handbook of community psychology, 2, 235-254. https:// doi.org/10.1037/14954-014

Sineace (2014). La acreditación en el Perú: Avances y perspectivas. Lima: Dirección de Evaluación y Acreditación - CONEAU

Ussher, M. (2008). La supervisión en la psicología social comunitaria. Revista Electrónica Internacional de la Unión Latinoamericana de Entidades de Psicología, (12), Recuperado de http://psicolatina. org/12/supervision.html

Velázquez, T., Rivera-Holguín, M. \& Custodio, E. (2017). Aportes y reflexiones de la formación en psicología comunitaria PUCP en tres regiones del Perú. Revista de Psicología, 35(1), 193-224. https://doi.org/10.18800/psico.201701.007

Velázquez, T., Rivera-Holguín, M. \& Custodio, E. (2015). El acompańamiento y el cuidado de los equipos en la Psicología Comunitaria: Un modelo teórico y práctico. Psicología, Conocimiento y Sociedad, 5(2), 307-334.

Velazquez, T. (2016). Universidad, ciudadanía y responsabilidad social: una relación necesaria. Recuperado de http://facultad.pucp.edu. pe/generales-letras/wp-content/uploads/2016/07/Leccion-inaugural-2016.pdf 
Vera, C. (2015). Dimensión ética en la formación de Psicología Comunitaria en universidades de Lima-Perú. Tesis para optar el título de Magíster en Psicología Comunitaria. Lima: Pontificia Universidad Católica del Perú.

Wiesenfeld, E. (2000). Entre la prescripción y la acción: la brecha entre la teoría y la práctica en las investigaciones cualitativas. FQS Forum: Qualitative Social Research, 1(2). http://www.qualitativeresearch.net/fqs-texte/2-00/2-00wiesenfeld-s.htm

Winkler, M., Velázquez, T., Rivera, M., Castillo, T., Rodríguez, A., Ayala, N. (2016). Ética y formación en Psicología Comunitaria: Análisis de programas y asignaturas en universidades latinoamericanas. Interamerican Journal of Psychology (IJP), 50(1), 23-31. https://journal.sipsych.org/index.php/IJP/article/view/78/pdf

Winkler, M. I. (2007). Cuestiones éticas en Psicología Comunitaria: dudas en la praxis. En J. Alfaro y H. Berroeta (Eds.), Trayectoria de la Psicología Comunitaria en Chile: Prácticas y Conceptos (pp. 373-400). Valparaíso: Universidad de Valparaíso.

Winkler, M. I., Alvear, K., Olivares, B. \& Pasmanik, D. (2010). Autonomia versus dependencia: quehacer comunitario y politicas públicas. Santiago de Chile: Universidad de Santiago de Chile. https:// doi.org/10.5027/psicoperspectivas-Vol13-Issue2-fulltext-353

Winkler, M. I., Alvear, K., Olivares, B. \& Pasmanik, D. (2014). Psicología Comunitaria hoy: Orientaciones éticas para la acción. Psicoperspectivas, 13(2), 43-54. 


\section{Apéndice 1. Análisis descriptivo de los sílabos}

\section{Tabla 1}

Análisis descriptivo de los sílabos $(n=20)$

\begin{tabular}{llc}
\hline Categorías & Subcategorías & Número \\
\hline Ubicación & Lima & 12 \\
& Otras regiones & 8 \\
\hline Tipo de universidad & Privada & 17 \\
& Nacional & 3 \\
\hline Créditos del curso & 2 & 4 \\
& 3 & 12 \\
& 4 & 3 \\
Mrabajo de campo & 8 & 1 \\
\hline & Obligatorio & 16 \\
& Electivo & 4 \\
\hline & Presente & 16 \\
& No se puede discernir & 3 \\
\hline
\end{tabular}

Recibido: 7 de julio, 2016

Revisado: 16 de agosto, 2017 Aceptado: 15 de diciembre, 2017 